\title{
The Vicarious Experiences Questionnaire(s): Online Tools for Measuring Mirror-Touch and Vicarious Pain
}

\author{
Mengze Li and Jamie Ward \\ School of Psychology, University of Sussex, Brighton UK \\ Contact: jamiew@sussex.ac.uk
}

\begin{abstract}
Vicarious experiences refer to shared feelings (e.g., emotion contagion) and sensations (e.g., pain or touch) that occur when an observer sees (or otherwise infers) that another person is in that state. There may be stable trait-level individual differences in this tendency. Here we report a dataset from two previously developed measures for vicarious touch and pain (Mirror-touch Questionnaire and Vicarious Pain Questionnaire), which we collectively term the Vicarious Experiences Questionnaire. As well as making this dataset publicly available, we also include a novel analysis of the data, together with tools for enabling other researchers to collect and analyse this data (video stimuli, an importable Qualtrics survey, and Excel sheets for classifying participants). We show that a subset of the 'normal' population experience reportable sensations of touch and pain when seeing it in others and demonstrate how they may be classified into several different groups. With regards to vicarious pain, we report separate 'sensorylocalised' and 'affective-general' groups (alongside non-responders) and consider the extent to which such differences are categorical or dimensional. For vicarious touch (or mirror-touch), we show that this tends to be found in people with a more extreme 'sensory-localised' profile for vicarious pain. The affective-general group has a stronger gender bias $(F>M)$ that is weaker in the sensory-localised group and absent for mirror-touch.
\end{abstract}

\section{INTRODUCTION}

Vicarious experiences refer to shared feelings (e.g., emotion contagion) and sensations (e.g., pain or touch) that occur when an observer sees (or otherwise infers) that another person is in that state. This is often regarded as a kind-of proto-empathy that emerges from the earliest stages of development (Decety \& Svetlova, 2012), or as a sub-component of empathy in its own right (often called affective empathy) that sits alongside other components involved in perspective-taking and mentalizing (inferences about the mental states of others) (Zaki \& Ochsner, 2012). To some extent, these different processes may be flexibly deployed such that certain instructions or perspectives enhance or diminish vicarious experiences. These can be measured, for example, in neuroimaging studies which show enhanced or diminished activity in brain regions that support those experiences (Lamm, Bukowski, \& Silani, 2016).

There may also be trait-level differences such that some people are more or less inclined to have vicarious experiences (when a given situation or context is matched across participants). The Emotion Contagion Questionnaire (Doherty, 1997) would be one such traitlevel measure. An example item is "If someone l'm talking with begins to cry, I get teary-eyed.". Others often use the Personal Distress Subscale of the IRI (Interpersonal Reactivity Index, (Davis, 1980)) to capture this, although it is to be noted that these items ask about emotional reactivity more broadly rather than shared (i.e., matched) responses. An example item is "I tend to lose control during emergencies".

Our own approach to measuring individual differences is to display videos of touch (Holle, Banissy, Wright, Bowling, \& Ward, 2011; Ward, Schnakenberg, \& Banissy, 2018) and 
pain (Grice-Jackson, Critchley, Banissy, \& Ward, 2017) to people and ask participants whether they report experiences of touch or pain on their own body. Most people report few if any such matched responses (although they might note offer kinds of response such as wincing or disgust). Those who do tend to report this can be described as having mirror-touch or mirror-pain synaesthesia because vision is triggering a somatosensory experience (Fitzgibbon et al., 2012), and this tendency is greater in people who also have more conventional types of synaesthesia (Ward, 2019). However, for present purposes nothing hinges on whether this is helpful or accurate to describe as a type of synaesthesia (Meier, Lunke, \& Rothen, 2015). All that matters is whether it is a stable and meaningful individual difference. The Vicarious Pain Questionnaire (VPQ) has been shown to have high test-retest reliability both at the level of individual scales and at the level of group membership (Botan et al., 2018). The test-retest reliability of the Mirror-touch Questionnaire (MTQ) is unknown, but both measures have good external validity extending over months and years. That is, group membership assessed on these questionnaires predicts performance on other questionnaires and cognitive tasks in subsequent sessions and predicts differences in brain structure and function in neuroimaging (Grice-Jackson et al., 2017; Ward et al., 2018). In effect, the tendency to report vicarious touch/pain is indicative of a broader profile of differences including social-emotional reactivity (Botan, Bowling, Banissy, Critchley, \& Ward, 2018) and body perception/awareness (Botan, Fan, Critchley, \& Ward, 2018; Bowling, Botan, Santiesteban, Ward, \& Banissy, 2019).

Both the VPQ and MTQ are somewhat unconventional in their presentation and scoring, and one of the current aims of is to make it easier for other researchers to adopt them. A secondary aim is to analyse and make available an unpublished dataset. In terms of presentation, both questionnaires involve the display of videos rather than just text-based questions and the responses require reporting phenomenology rather than Likert-scale answers. For example, in the MTQ participants may give answers such as 'nonpainful touch', 'tingling', 'itchiness', 'pain without touch' (Ward et al., 2018). In the VPQ, participants reflect on the nature of any vicarious pain response such as whether the pain is localised or generalised, and select from sensory and affective descriptors such as 'sharp' or numb' (GriceJackson et al., 2017). In terms of analysis, the number of critical responses is totalled. For MTQ, this corresponds to the number of tactile responses elicited from seeing a human touched (a score out of 14). Ward et al. (2018) argue that a score of 7 or above should be taken as a cut-off based on its external validity against other measures (e.g., emotional reactivity, facial expression recognition). They report that $2.1 \%$ of an undergraduate sample meet that cut-off. For the VPQ, a data-driven approach was used to identify groups based on a cluster analysis. Here the data consists of a measure of the amount of vicarious pain either the total number of pain responses (out of 16 videos) or the average pain intensity across those videos - together with two further dimensions related to the quality of vicarious pain: number of sensory minus affective descriptors (S-A) and the number of localised minus generalised responses (L-G). The cluster analysis identified three groups. Non-responders, the majority of people, have scores close to zero on all dimensions. There were two groups who reported high amounts of vicarious pain: a group who tends to use sensory descriptors and has localised pain (SL group) and a group who tends to use affective descriptors and reports generalised pain (AG group). Grice-Jackson et al. (2017) report a prevalence of $16.8 \%$ and $10.4 \%$ for the latter two groups.

The use of a cluster analysis in the VPQ has the advantage of being data-driven (e.g., leading to the discovery of two rather than one responder groups). But it may also be a barrier to others adopting the measure because of the unfamiliarity of the technique and the fact that it requires large samples to run one. However, by making available a normative sample (from nearly 2000 people) other researchers can make use of our dataset to classify any new individual. Classification is merely based on the shortest distance to one of the three cluster centroids (averages). For example, if a person's score is closest to the SL centroid then that person is classed as SL. This is illustrated in Figure 1. We create an excel calculator for doing this (but it is simple enough to calculate with a pocket calculator). A cluster analysis works according to the exact same principle - participant classification is dictated by the nearest cluster centroid - but with the added difference that the cluster centroids are recalculated 
every time a new person is added to the sample. When the sample is sufficiently large then adding new people will have little impact so one can treat the centroids as stable.

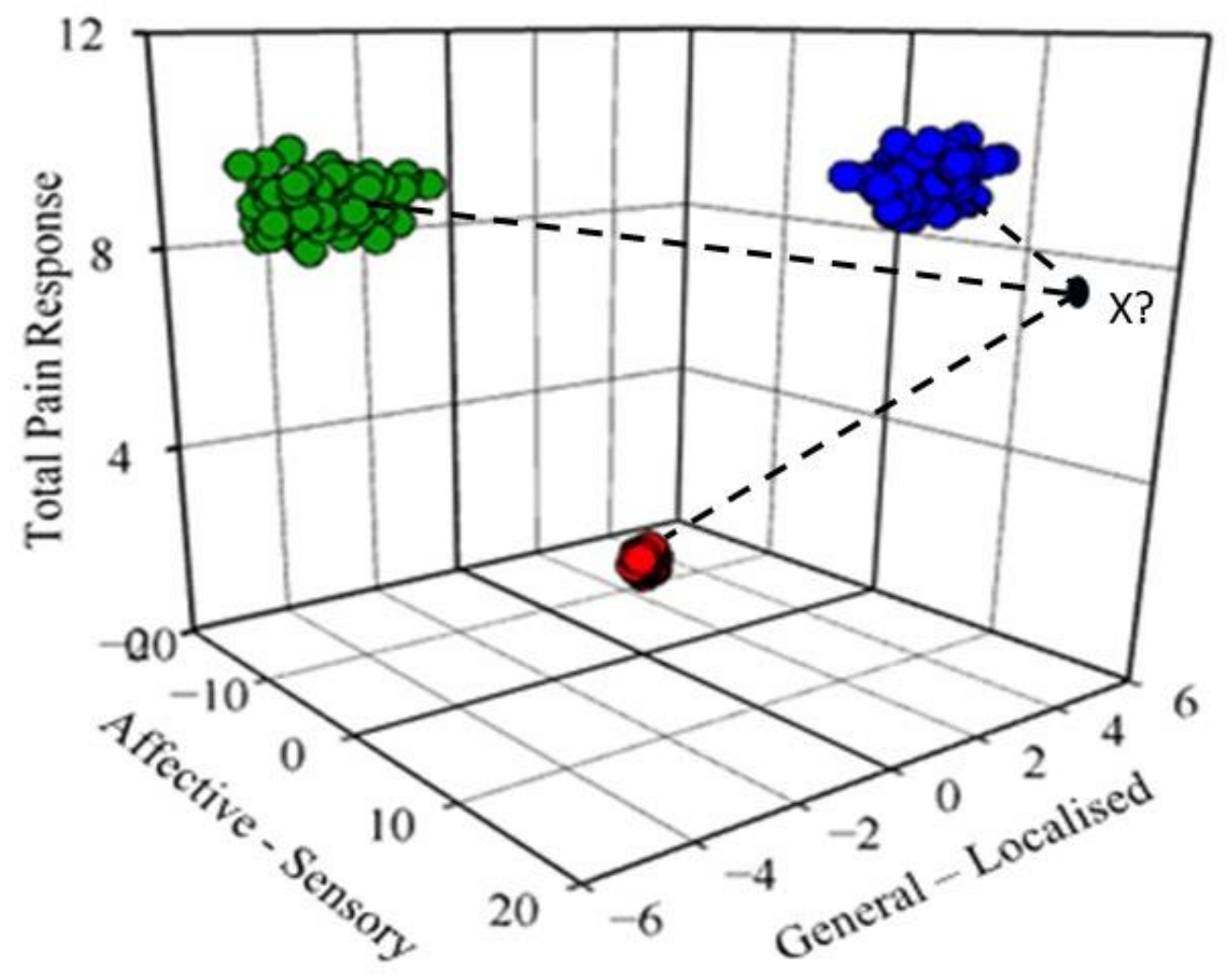

Figure 1. An unclassified participant $(X)$ given the VPQ can be classified by measuring the shortest distance to a cluster centroid (red = non-responder, blue $=S L$, green $=A G$ ). In this example, the participant is closest to the blue SL centroids (note that the actual distance calculations must be made on z-scores rather than raw distances). In this figure, bootstrapped sampling was performed on $\mathrm{N}=100$ participants (sampled with replacement from a wider pool of $N=573$ ) such that different coloured dots represent different centroid estimates (not different participants). Centroids are relatively stable even for this small sample size. In the present study, we have a much larger sample $(N=1972)$ and calculate fixed centroids (without bootstrapping) for others to make use of. Figure adapted from Grice-Jackson et al. 2017.

\section{METHOD}

\section{Participants}

The VPQ dataset. After removal of duplicates, the dataset consists of $N=1972$ participants who took this measure between October 2018 and April 2021. The mean age was 20.579 years (S.D. $=4.736$; note that age is missing for 34 participants) and there were 1543 females, 416 males, and 13 as other/non-binary. These were recruited as an opportunistic sample of psychology undergraduates from the University of Sussex who took the measure for course credits. There is no overlap with the samples reported previously by Grice-Jackson et al. (2017) or Botan et al. (2018).

The MTQ dataset. This is a subset of $\mathrm{N}=1425$ participants from the VPQ dataset who were asked to complete the MTS questionnaire of Ward et al. (2018), minus the pain items, in the same session as the VPQ. The mean age was 20.066 years (S.D. $=3.833$ ) and there 
were 1135 females, 281 males, and 9 as other/non-binary. None of these participants were previously reported by Ward et al. (2018).

The research was approved by the Cross-Schools Science and Technology Research Governance and Ethics Committee of the University of Sussex, UK.

\section{Materials and Procedure}

The video stimuli are uploaded to a separate folder in this OSF repository and are also available on youtube at

https://www.youtube.com/channel/UCT8goTgWGRsu14NjVaPCSGw/videos (VPQ) and https://www.youtube.com/channel/UC IwG3ScoCR8EU9m fPe6BA (MTQ). All data was collected using Qualtrics and a script for importing into this software is also available in the OSF repository.

The VPQ consists of 16 video clips depicting mild-moderate pain, either injections or sporting injuries (Grice-Jackson et al., 2017). Each clip was around 10 seconds long and had no audio. After each clip, participants were initially asked whether they experienced a sensation of pain in their own body when viewing the video (yes/no) and were asked to rate the unpleasantness ("How unpleasant did you find the experience of watching this video? 1= not unpleasant, $10=$ highly unpleasant"). If participants answered 'yes' to the first question they were asked three further questions: (1) how intense their pain experience was (1= very mild, $10=$ intense pain; 0 was used to code people who reported no pain at all); (2) to indicate the location of their experience (either 'localised to the same point as the observed pain', 'localised but not to the same point, and 'a general/ non-localisable pain experience'); and (3) to select pain descriptors that best describe their experience (from a set of 20 with multiple selections possible). The latter were obtained from a standard set used in the pain assessment literature (Melzack, 1975) and comprised 10 descriptors with sensory qualities (e.g. "sharp") and 10 descriptors with affective qualities (e.g. "nauseating").

The MTQ consisted of 24 short video clips depicted touch and itch. There were fourteen videos depicting touch to a human (from Holle et al., 2011), and these are the stimuli that are scored to determine participant classification. These comprised of 7 pairs of stimuli depicting touch to either the left or right, namely: 1) touch to the cheek with a finger; 2) touch to the cheek with the tip of a knife; 3 ) touch to the hands in egocentric perspective with a finger; 4) touch to the hands in egocentric perspective with a knife; 5) touch to the hands in allocentric perspective with a finger; 6 ) touch to crossed hands in egocentric perspective with a finger; 7 ) touch to the cheek with a finger with face inverted. In all cases, the model was a Caucasian female and hands were palm down. Additionally, there were six videos of touch to inanimate objects namely a dummy head, dummy hands (egocentric perspective), and a fan - both from the left and right side (from Holle et al., 2011). There were four videos, 20s long, depicting someone scratching intensely their chest or upper arm (from Holle, Warne, Seth, Critchley, \& Ward, 2012). After each video was played participants were asked: "Do you experience anything on YOUR body? (excluding feelings of unease, disgust, or flinching) [Yes/No]". Upon an affirmative answer they were asked three follow-up questions. Firstly, they were asked "How would you describe the sensation?" and they were forced to choose one from the following checkbox options: Touch (without pain); Pain (without touch); Painful touch; Tingling; Itchiness; Feeling of being scratched; and Other [Please specify_]. Secondly, they were asked "Where on YOUR body was it felt?" and they were forced to choose from one of the following options: Not localisable; Left face; Right face; Left hand; Right hand; Left arm; Right arm; Chest; Back; other [Please specify__ . Finally, they were asked: "How intense was it? $(0=$ nothing, $10=$ very intense)".

\section{Data Analysis: VPQ}

A video demonstration as to how to perform the cluster analysis of the VPQ using SPSS is provided on youtube (https://www.youtube.com/watch?v=iYkVmLfAt6w). Please note that the clustering should be performed on z-scores in both steps of the analysis, and we recommend z-transforming the data before copying into SPSS (noting that the video did not 
use z-scores in the second step by error; the actual difference in classification is small). A cluster analysis was used to group participants based on their responses to a number of input variables from the VPQ. The input variables used were mean intensity of pain experiences, the number of localised responses minus the number of generalized responses, and the number of sensory descriptors minus the number of affective descriptors. All three variables were z-transformed prior to analysis. A two-step cluster analysis was performed (Zhang, Ramakrishnan, \& Livny, 1996) which initially involved a hierarchical cluster analysis using Ward's (1963) method followed by a non-hierarchical k-means analysis with 50 iterations. The cluster centroids and number of clusters for the k-means analysis were guided by the hierarchical analysis.

The classification of all participants in the dataset is based on the closest cluster centroid (Euclidean distances based on z-scores). Similarly, one can apply the same principle to any new participant without rerunning the cluster analysis. That is, one can assume that (beyond a certain sample size) the clustering algorithm is sufficiently stable that adding new participants does not induce a shift in the solution. This means that other research groups can use this classification scheme without performing any cluster analysis (which would require several hundred participants). An attached excel sheet calculator performs the vicarious pain classification when the researcher enters the three input variables (mean pain intensity, sensory minus affective score, localised minus general score). It does so by transforming the data into z-scores and finding the nearest cluster centroid. Whilst this has the advantage of providing harmonization across research groups, there is also some need for caution. For example, if the sensory and affective pain descriptors are not cross-culturally appropriate then scores obtained elsewhere may not align to the English-language norms of our sample.

\section{Data Analysis: MTQ}

For identifying mirror-touch synaesthesia, we are only interested in tactile sensations when observing humans and, for this purpose, the categories of touch (without pain), painful touch, and the feeling of being scratched were combined along with 'other' responses that described touch (e.g. "pressure" being the most common term). Thus, participants receive a score between 0 and 14 and with a score of 7 or above being indicative of MTS.

\section{RESULTS}

\section{Cluster Analysis of the VPQ}

Table 1 shows the number of participants in each of the three clusters and their demographic characteristics, as well as the cluster centroids (both raw scores and ztransformed). Considering only binary genders (i.e., those participants identifying as male or female), the prevalence of SL responders amongst women was $13.74 \%$ versus $11.29 \%$ amongst men (1.22-to-1 female:male ratio). The prevalence of AG responders amongst women was $13.48 \%$ versus $7.21 \%$ amongst men (1.87-to- 1 female:male ratio). That is, vicarious pain responders are more likely to be female and this is driven most strongly by the AG group (comparing the three clusters: $x^{2}(2)=15.406, p<.001$ ). Overall, the estimated population prevalence for $S L$ and $A G$ responders is $12.51 \%$ and $10.34 \%$ respectively (these being somewhat lower than the observed prevalence rates from our own sample, $13.29 \%$ and $11.97 \%$, which are skewed by having a higher proportion of women). The figures from GriceJackson et al. (2017) were $16.8 \%$ and $10.4 \%$ based on an independent sample and a slightly different analysis method (which used total pain responses rather than mean intensity of pain responses).

Table 1: Results of a two-step hierarchical and k-means cluster analysis that extracts three groups: Non-responders, and two types of vicarious pain responders termed $S L$ (sensory/localised) and AG (affective/general). 


\begin{tabular}{|c|c|c|c|c|c|c|c|c|}
\hline \multicolumn{3}{|c|}{ Sample details } & \multicolumn{3}{|c|}{ VPQ scores (mean, S.D.) } & \multicolumn{3}{|c|}{$\begin{array}{l}\text { Cluster centroids } \\
\text { (z-scores) }\end{array}$} \\
\hline Cluster & $\mathbf{N}$ & F:M:Other & $\begin{array}{l}\text { Pain } \\
\text { intensity }\end{array}$ & S-A & L-G & $\begin{array}{l}\text { Pain } \\
\text { intensity }\end{array}$ & S-A & L-G \\
\hline $\begin{array}{l}\text { Non- } \\
\text { responders }\end{array}$ & 1474 & 1123:339:12 & $\begin{array}{l}0.327 \\
(0.525)\end{array}$ & $\begin{array}{l}0.588 \\
(2.700)\end{array}$ & $\begin{array}{l}0.341 \\
(1.608)\end{array}$ & -0.4501 & -0.092 & -0.0077 \\
\hline $\begin{array}{l}\text { SL } \\
\text { responders }\end{array}$ & 260 & $212: 47: 1$ & $\begin{array}{l}3.004 \\
(1.645)\end{array}$ & $\begin{array}{l}11.031 \\
(8.149)\end{array}$ & $\begin{array}{l}5.331 \\
(4.039)\end{array}$ & 1.4248 & 1.4295 & 1.4334 \\
\hline $\begin{array}{l}\mathrm{AG} \\
\text { responders }\end{array}$ & 238 & 208:30:0 & $\begin{array}{l}2.727 \\
(1.439)\end{array}$ & $\begin{array}{l}-5.563 \\
(10.924)\end{array}$ & $\begin{array}{l}-4.891 \\
(3.322)\end{array}$ & 1.2312 & -0.989 & -1.5183 \\
\hline
\end{tabular}

Figure 2 shows the frequency distribution of responder scores (as a 2D density plot) on the S-A and L-G axes of the VPQ. It can be seen that there are two 'hot spots' where people are likely to cluster that contain the group means (centroids) for the AG and SL groups. However, it is also to be noted that there is no hard boundary between the categories and there would be a low degree of confidence in the classification of participants with scores close to the classification boundary (i.e., equidistant to centroids). Researchers may either choose to omit such participants (according to some predefined criteria) or accept some degree of noise in the creation of groups (i.e., such that groups broadly, but not exactly, correspond to the categories of non-responder, AG, and SL). Our approach to date has been the latter. A third approach would be to create a continuous dimension with endpoints of SL and AG: i.e., such that participant's scores are projected onto a line at 90 degrees to the classification boundary (such a line would capture the most variance amongst responders). In effect, instead of studying three groups one would split the participants into two groups (responders and non-responders) and then apply a continuous individual differences metric within the responder group themselves. The equation for this line can be computed from knowing the two centroids (in z-space) of the AG and SL groups in Table 1 and is:

$[S-A]=0.819[L-G]+0.255$

The slope is calculated as $=(1.4295+0.989) /(1.4334+1.5183)=0.819$

The intercept is given by $=1.4295-\left(\right.$ slope $\left.^{*} 1.4334\right)=0.255$

Individual datapoints can be projected on to this line and re-centred such that the midpoint of the centroids acts as the origin of the line with positive numbers representing distances in the $S L$ direction and negative numbers representing distances in the AG direction. The distribution of scores along that axis is shown in Figure 3. Whether this approach accounts for the data better than a categorical approach is something for future research to explore. 


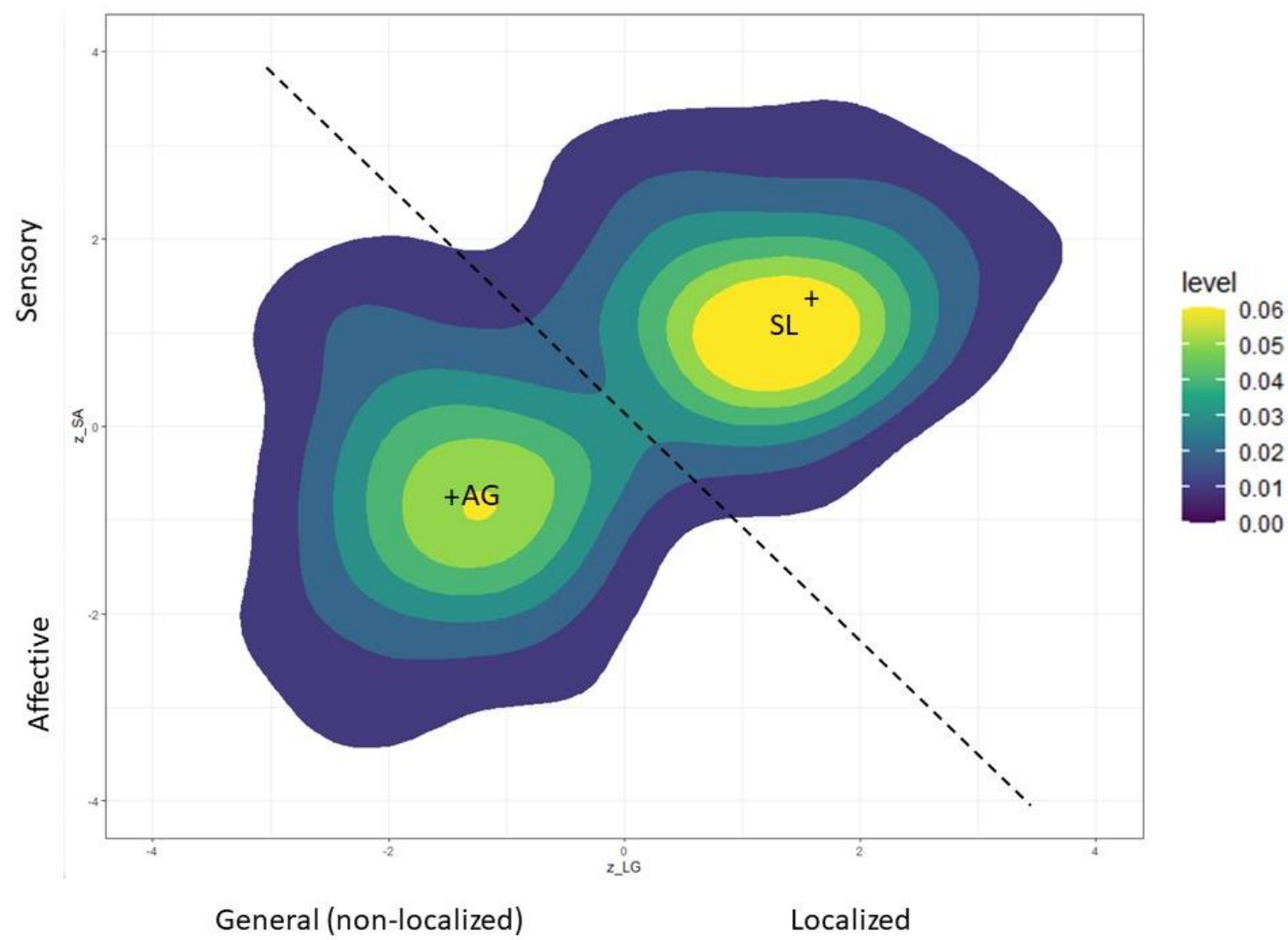

Figure 2: The 'phenomenological space' of vicarious pain as illustrated by a density plot of all vicarious pain responders ( $A G$ and $S L$ ) on the $S-A$ and $L-G$ axes. Colors represent differences in density levels (with yellow being the highest). The plus sign (+) shows the means of the groups (from Table 1) and the dotted line shows the approximate position of the classification boundary (noting that individual participants are classified according to their closest distance to the group mean). 


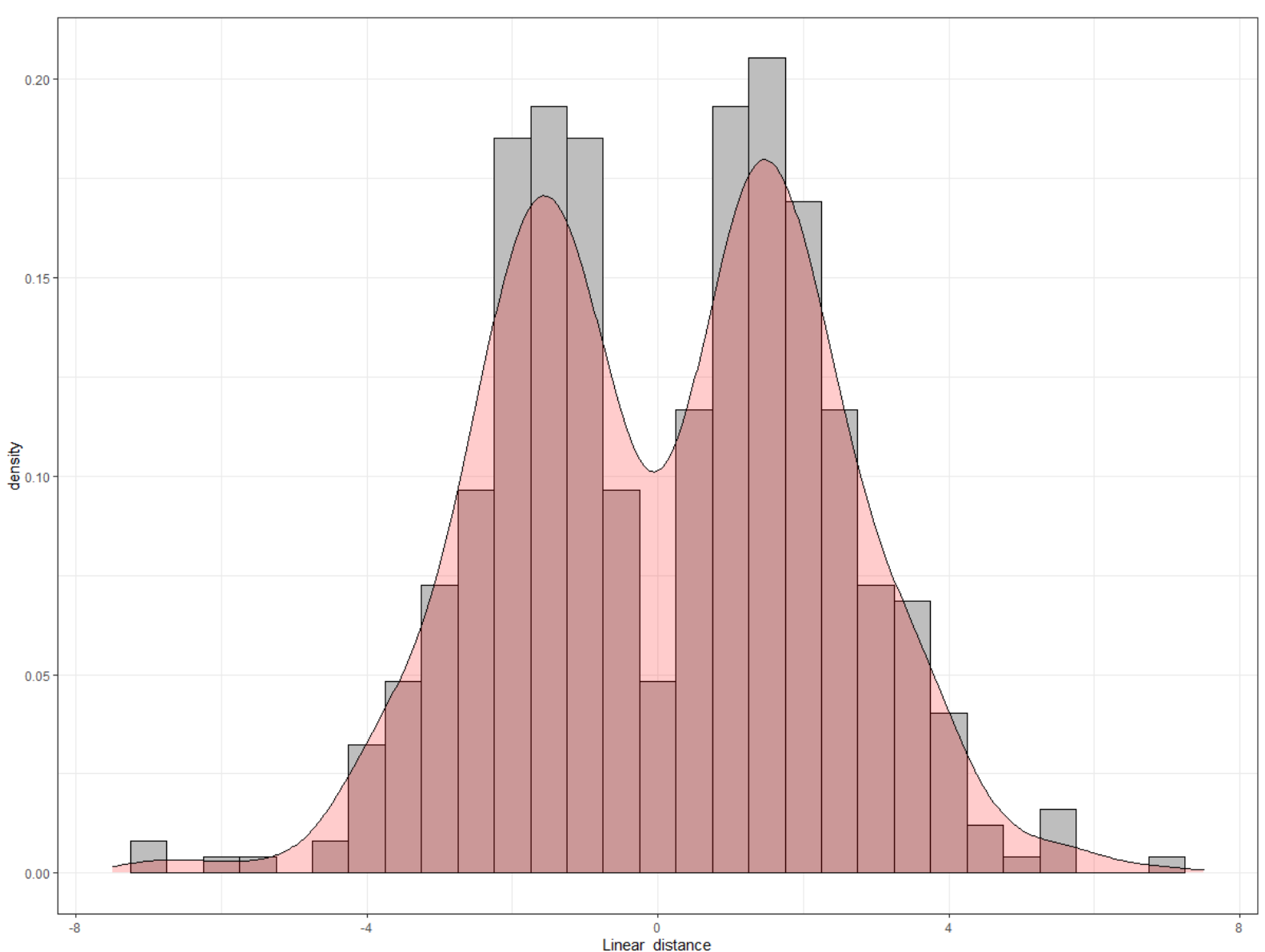

Figure 3: Histogram (grey) and density plot (pink) for vicarious pain responders (both SL and $A G)$ projected on to a linear dimension which is the line of best fit between the two centroids of those groups.

\section{Unpleasantness scores for the VPQ}

The unpleasantness ratings of the pain videos are not entered into the cluster analysis and, therefore, could be regarded as an independent test of the validity of those groupings. Grice-Jackson et al. (2017) reported this for a number of items including unpleasantness, the presence of vicarious pain in daily life, and self-reported empathy. Here, we replicate those results with regards to a significant group difference in unpleasantness ratings $(F(2,1971)=$ $36.595, p<.001)$ with the non-responder group having lower scores $(4.450$, S.D. $=1.635)$ than either the SL group (5.137, S.D. = 1.610) or the AG group (5.195, S.D. = 1.479).

\section{Relationship between MTS and VPQ}

The prevalence of MTS in men was $5.34 \%$ (15/281) and in females was $6.41 \%$ $(73 / 1139)$ which was not significantly different $\left(x^{2}(1)=.445, p=.505\right)$. Across the three clusters identified in the analysis of the VPQ, the prevalence of MTS was $2.56 \%(28 / 1092)$ amongst non-responders, 26.06\% (49/188) amongst the SL responders, and 7.58\% (11/145) amongst $A G$ responders. This was significantly different $\left(X^{2}(2)=153.421, p<.001\right)$. Figure 4 shows the phenomenological profile of people with MTS on the VPQ (irrespective of cluster membership). This shows a single dominant cluster primarily (although not exclusively) within the sensory and localised quadrant.

There is evidence that, even within the SL cluster, people who additionally report MTS are more extreme in their vicarious pain responses. The average intensity to pain stimuli by SL responders who also have MTS was $3.908(S D=1.905)$ compared to $2.755(S D=1.573)$ for $S L$ responders who don't report MTS $(t(186)=4.170, p<.001$, Cohen's $d=.703)$. They also reported more sensory than affective descriptors (13.3676, S.D. $=9.701$ compared to 10.626 , S.D. $=7.784 ; \mathrm{t}(186)=1.983, \mathrm{p}=.049$, Cohen's $d=.356)$. The localised minus generalized 
score did not differ between the subgroups (6.694, S.D. $=4.297$ compared to 5.389 , S.D. $=$ $4.189 ; \mathrm{t}(188)=1.863, \mathrm{p}=.064$, Cohen's $\mathrm{d}=.320)$.

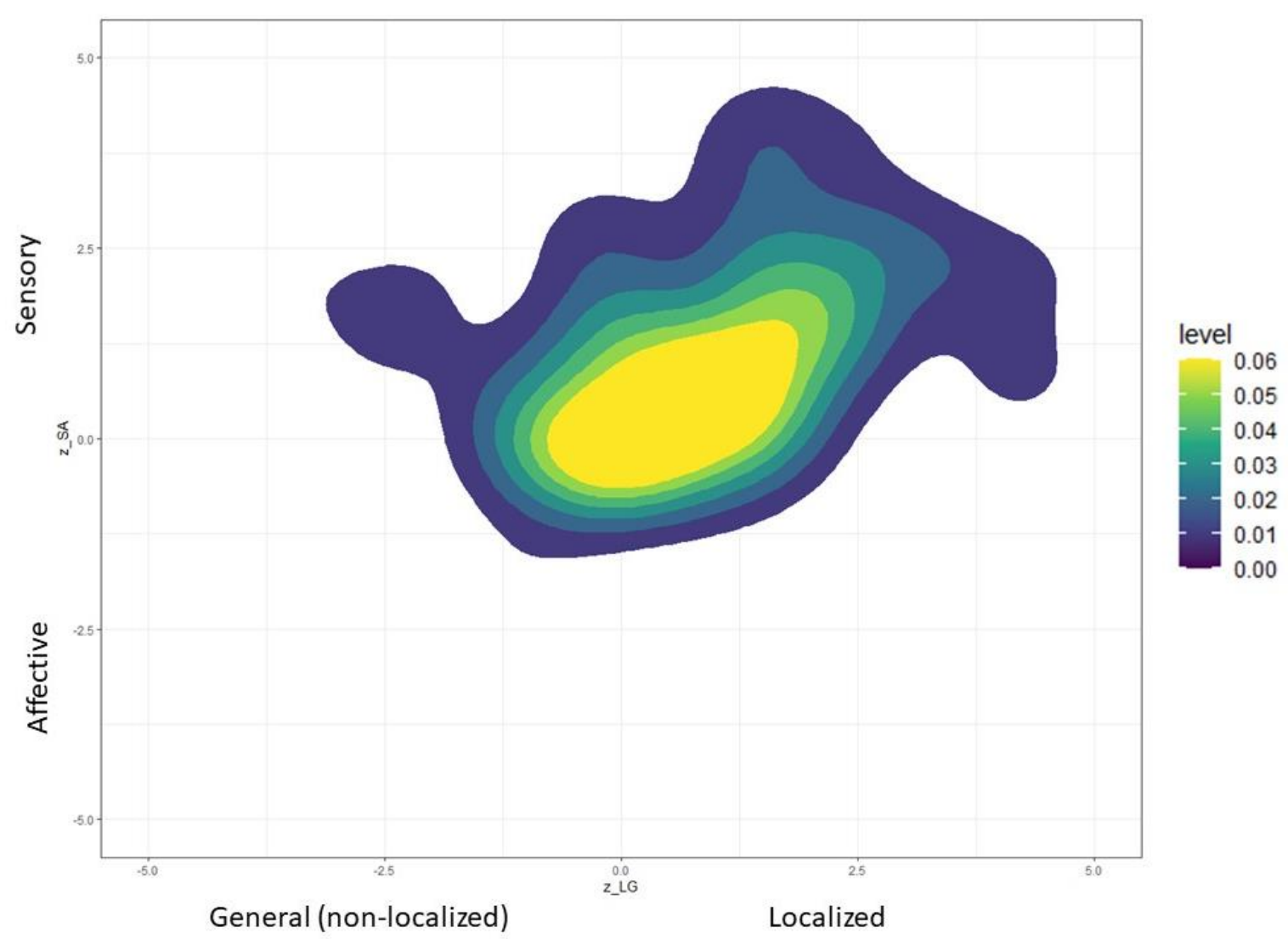

Figure 4: The VPQ scores of participants classified as having mirror-touch synaesthesia projected on to the phenomenological space defined by the S-A and L-G axes. Yellower colours represent high density of participants in that region. Note that scores tend to fall in the sensory + localized quadrant.

\section{DISCUSSION}

One of the main aims of this study was to provide new data and share analysis tools for the study of vicarious experiences of touch and pain. There exists, within the 'normal' population, a non-trivial minority of people who consciously report experiencing pain and touch when seeing (or inferring) that someone else is in that state. This is a relatively stable trait that persists over time. The differences may not be limited to touch/pain but could extend to differences in social and emotional cognition (Botan, Bowling, et al., 2018) and differences in the computation of self-other boundaries e.g. in experimental studies of body ownership (Botan, Fan, et al., 2018). In effect, these kinds of vicarious experiences may be indicative of broad suite of differences that mutually influence each other and presumably contribute to its development. Although we treat these differences as categorical, it is an empirical question as to whether this is the optimal approach. However, a single continuous scale (from low to high) is unlikely to be satisfactory in the case of vicarious pain because of the qualitative differences between AG and SL groups (who are both high but have a bimodal profile). We provide tools to enable other researchers to categorise individual participants without having to run complex statistics (such as cluster analysis).

The study also provides new information on the prevalence and gender difference of these vicarious experiences. Within the same sample, we find that the gender difference for 
the AG group (1.87: 1, in favour of women) is larger than for the SL group and people with mirror-touch (both $1.2: 1$ ). Mirror-touch tends to be rarer and is more likely to be found in the SL group and, in particular, those with more extreme scores within that group. As well as being co-morbid there is a similar phenomenology (mirroring of body locations as well as sensations) and, likely, a similar cause. In terms of generalizing our findings, we note that our data is collected from a WEIRD sample - Western, Educated, Industrialised, Rich, Democratic (Henrich, Heine, \& Norenzayan, 2010). The extent to which the results will be comparable in other cultures, such as those with different conceptualisations of pain and "somatization" of mental health needs to be explored. Nevertheless, we suggest that within cultures there are likely to be some individuals with trait-like tendencies towards conscious vicarious experiences against a background of others with little or no tendency in this regard.

Finally, it is important to note that our approach is neither the only one nor the first. Osborn and Derbyshire (2010) were the forerunners of our approach - they presented a series of images (e.g., of serious sports injuries) and participants who reported pain in themselves to one or more of these were classed as responders (and shown to differ in their neuroimaging response to observing pain). The Empathy for Pain Scale takes a similar approach to the VPQ in its use of video stimuli (e.g., of a surgical procedure) and phenomenological ratings of reactions (Giummarra et al., 2015). Their ratings consisted of 12 descriptors which fell into three factors which they termed Affective Distress (such as, "visceral sensations (e.g. nausea)"), Empathic Concern (e.g. "sense of compassion") and Vicarious Pain (e.g. "painful sensations"). We assume that the third subscale relates to our VPQ groups, although it is possible that the AG group is linked to Affective Distress. It would be important for future research to address this by directly comparing measures. When their three Empathy for Pain subscales are combined with anxiety measures in a cluster analysis (similar to our approach) then two groups with high empathy for pain emerge (one with high anxiety and one with low anxiety), together with a third group with low empathy for pain and low anxiety (Nazarewicz, Verdejo-Garcia, \& Giummarra, 2015). In this study, the three Empathy for Pain subscales tended to group together rather than fractionate. Although we do now know how these alternatives map on to the SL and AG groups that we have studied, it is certainly possible that the 'phenomenological space' extends beyond those dimensions tapped by the VPQ.

\section{References}

Botan, V., Bowling, N. C., Banissy, M. J., Critchley, H., \& Ward, J. (2018). Individual Differences in Vicarious Pain Perception Linked to Heightened Socially Elicited Emotional States. Frontiers in psychology, 9. doi:10.3389/fpsyg.2018.02355

Botan, V., Fan, S., Critchley, H., \& Ward, J. (2018). Atypical susceptibility to the rubber hand illusion linked to sensory-localised vicarious pain perception. Consciousness and Cognition, 60, 62-71. doi:10.1016/j.concog.2018.02.010

Bowling, N. C., Botan, V., Santiesteban, I., Ward, J., \& Banissy, M. J. (2019). Atypical bodily self-awareness in vicarious pain responders. Philosophical Transactions of the Royal Society B-Biological Sciences, 374(1787). doi:10.1098/rstb.2018.0361

Davis, M. H. (1980). A multi-dimensional approach to individual differences in empathy. JCAS Catalog of Selected Documents in Psychology, 75, 989-1015.

Decety, J., \& Svetlova, M. (2012). Putting together phylogenetic and ontogenetic perspectives on empathy. Developmental Cognitive Neuroscience, 2(1), 1-24. doi:10.1016/j.dcn.2011.05.003

Doherty, R. W. (1997). The emotional contagion scale: A measure of individual differences. Journal of Nonverbal Behavior, 21(2), 131-154. Retrieved from <Go to ISI>://A1997XG54400003

Fitzgibbon, B. M., Enticott, P. G., Rich, A. N., Giummarra, M. J., Georgiou-Karistianis, N., \& Bradshaw, J. L. (2012). Mirror-sensory synaesthesia: Exploring 'shared' sensory experiences as synaesthesia. Neuroscience and Biobehavioral Reviews, 36(1), 645657. doi:10.1016/j.neubiorev.2011.09.006 
Giummarra, M. J., Fitzgibbon, B. M., Georgiou-Karistianis, N., Beukelman, M., VerdejoGarcia, A., Blumberg, Z., . . Gibson, S. J. (2015). Affective, sensory and empathic sharing of another's pain: The Empathy for Pain Scale. European Journal of Pain, 19(6), 807-816. doi:10.1002/ejp.607

Grice-Jackson, T., Critchley, H. D., Banissy, M. J., \& Ward, J. (2017). Common and distinct neural mechanisms associated with the conscious experience of vicarious pain. Cortex, 94, 152-163.

Henrich, J., Heine, S. J., \& Norenzayan, A. (2010). The weirdest people in the world? Behavioral and Brain Sciences, 33(2-3), 61-+. doi:10.1017/s0140525x0999152x

Holle, H., Banissy, M., Wright, T. D., Bowling, N., \& Ward, J. (2011). "That's not a real body": Identifying stimulus qualities that modulate synaesthetic experiences of touch. Consciousness and Cognition, 20, 720-726.

Holle, H., Warne, K., Seth, A. K., Critchley, H. D., \& Ward, J. (2012). The neural basis of contagious itch and why some people are more prone to it. Proceedings of the National Academy of Science, USA, 109, 19816-19821.

Lamm, C., Bukowski, H., \& Silani, G. (2016). From shared to distinct self-other representations in empathy: evidence from neurotypical function and socio-cognitive disorders. Philosophical Transactions of the Royal Society B-Biological Sciences, 371(1686). doi:10.1098/rstb.2015.0083

Meier, B., Lunke, K., \& Rothen, N. (2015). How mirror-touch informs theories of synesthesia. Cognitive Neuroscience, 6(2-3), 142-144. doi:10.1080/17588928.2015.1057484

Melzack, R. (1975). The McGill Pain Questionnaire: major properties and scoring methods. Pain, 1(3), 277-299.

Nazarewicz, J., Verdejo-Garcia, A., \& Giummarra, M. J. (2015). Sympathetic pain? A role of poor parasympathetic nervous system engagement in vicarious pain states. Psychophysiology, 52(11), 1529-1537. doi:10.1111/psyp.12516

Osborn, J., \& Derbyshire, S. W. G. (2010). Pain sensation evoked by observing injury in others. Pain, 148, 268-274.

Ward, J. (2019). The Co-occurrence of Mirror-Touch With Other Types of Synaesthesia. Perception, 48(11), 1146-1152. doi:10.1177/0301006619875917

Ward, J., Schnakenberg, P., \& Banissy, M. J. (2018). The relationship between mirror-touch synaesthesia and empathy: New evidence and a new screening tool. Cognitive Neuropsychology, 35, 314-332.

Ward, J. H. (1963). Hierarchical grouping to optimize an objective function. Journal of the American Statistical Association, 58(301), 236-244.

Zaki, J., \& Ochsner, K. (2012). The neuroscience of empathy: progress, pitfalls and promise. Nature Neuroscience, 15(5), 675-680. doi:10.1038/nn.3085

Zhang, T., Ramakrishnan, R., \& Livny, M. (1996). BIRCH: An Efficient Data Clustering Method for Very Large Databases. SIGMOD Record, 25(2), 103-114 